\title{
Mandating ultrasound usage for internal jugular vein cannulation
}

\author{
Steven M. Neustein, MD
}

Received: 18 May 2010/Accepted: 11 June 2010/Published online: 13 July 2010

(C) Canadian Anesthesiologists' Society 2010

\section{To the Editor,}

Dr. Ayoub et al. have published a thorough and excellent review of the use of ultrasound for cannulation of the internal jugular vein. ${ }^{1}$ Both the incidence of complications and the failure rate were found to be reduced significantly with the use of ultrasound. ${ }^{1}$ We have experienced similar results in our department. In fact, our medical board considers the role of ultrasound for internal jugular vein cannulation to be of such importance that they adopted a policy to use ultrasound for the placement of all internal jugular vein catheters. We also have an additional departmental policy stipulating that the position of the initial catheter must be checked by manometry prior to converting it to an indwelling central catheter. Manometry can be accomplished by attaching sterile tubing to the catheter, filling the tubing with blood, and then elevating the tip of the tubing such that the blood drains back into the vessel, which it will do if accessing the jugular vein.

In their conclusion, Dr. Ayoub et al. recommend that ultrasound be used, but they do not discuss the role of either the department or the hospital in achieving this goal. We recommend that the physicians ask the hospital to purchase an adequate number of ultrasound devices to facilitate using ultrasound for all central venous access procedures. Once there is a sufficient supply of ultrasound devices and they are put to use, a policy can be established. Thus, the adoption of this technique becomes a systems approach, rather than solely the individual practitioner's choice to utilize the procedure. Placing central lines during an emergency should be excluded from this policy.

S. M. Neustein, MD ( $\square)$

Mount Sinai Medical Center, New York, NY, USA

e-mail: steve.neustein@mountsinai.org
Funding No funding sources.

\section{Reference}

1. Ayoub $C$, Lavallee $C$, Denault A. Ultrasound guidance for internal jugular vein cannulation: Continuing Professional Development. Can J Anesth 2010; 57: 500-14.

\section{Reply}

We welcome the thoughtful comments by Dr. Neustein on our Continuing Professional Development module, and we do share the same enthusiasm regarding the use of ultrasound for central vein access. Properly used, ultrasound guidance increases success rate and minimizes complications, provided that the operator remains conscious of the limitations of the technique and is aware of its potential hazards if not properly employed. The benefits of ultrasound are directly related to proper training. We agree with McGee and Gould who conclude that the use of ultrasound guidance on a routine basis should be considered when ultrasound equipment is available and physicians have adequate training. ${ }^{1}$

Regarding manometry, we recommend connecting the initial catheter to pre-flushed sterile tubing linked to a pressure transducer so that the actual central venous pressure tracing can be displayed on the scope. This technique limits blood spill and air penetration into the system. We do not use manometry on a routine basis in our institution. We prefer following the position of the guidewire with our transducer all the way down to the innominate vein close to its junction with the superior vena cava. In addition, as we are performing mainly cardiac surgery, we routinely use transesophageal echocardiography (TEE) as recommended 
by the recent guidelines. ${ }^{2}$ The TEE probe is inserted after the induction of anesthesia before central venous catheterization. Using a mid-esophageal bicaval position, the appearance of the guidewire in the superior vena cava can be confirmed easily before the final insertion of the catheters. Presence of the guidewire in the superior vena cava rules out any arterial position. This approach is used in several centres that do not even use echo-guided vascular access. However, using TEE for confirmation would not be practical in non-intubated patients.

The cost of acquiring ultrasound machines remains a limitation for hospital administrators. However, several companies now produce echocardiographic equipment, and we have observed a gradual decrease in the price of the equipment since the early 1990s.
Competing interests None declared.

\section{References}

1. McGee DC, Gould MK. Preventing complications of central venous catheterization. N Engl J Med 2003; 348: 1123-33.

2. Anonymous. Practice Guidelines for Perioperative Transesophageal Echocardiography: An Updated Report by the American Society of Anesthesiologists and the Society of Cardiovascular Anesthesiologists Task Force on Transesophageal Echocardiography*. Anesthesiology 2010; 112: 1-13.

Christian Ayoub, MD

André Denault, $\mathrm{MD}, \mathrm{PhD}$

University of Montreal, Montreal, QC, Canada

e-mail: cr.ayoub@umontreal.ca 